\title{
Exploring quantum dynamics in an open many-body system: Transition to superradiance
}

\author{
Alexander Volya $\nmid$ and Vladimir Zelevinsky $\ddagger$ \\ $\dagger$ Physics Division, Argonne National Laboratory, Argonne, Illinois 60439 \\ $\ddagger$ National Superconducting Cyclotron Laboratory and Department of Physics and \\ Astronomy, Michigan State University, East Lansing, Michigan 48824
}

\begin{abstract}
We study the dynamics of a complex open quantum many-body system. The coupling to external degrees of freedom can be viewed as a coupling to a radiation field, to continuum states or to a measuring apparatus. This perturbation is treated in terms of an effective non-Hermitian Hamiltonian. The influence of such coupling on the properties of the many-body dynamics is discussed, with emphasis on new effects related to dynamical segregation of fast and slow decays and the phase transition to Dicke superradiance. Relations to quantum optics, continuum shell model, theory of measurement, quantum chaos, percolation theory, and to quantum reactions are stressed.
\end{abstract}




\section{Introduction}

The interplay of complex intrinsic dynamics with radiation, coupling to the continuum or to any other external influence, such as measurement, is an important subject for quantum optics, nanoscale devices, theory of decoherence and quantum complexity, future quantum computing, and physics of nuclei far from stability, to name just few branches of modern physics, seemingly far remote from each other. After all, the complete isolation of a "system" and the "external world" is never possible. The chaotic and regular aspects of many-body physics, as well as openness and decay properties, are deeply rooted in valuable parts of Wigner's legacy. In this paper, commemorating Wigner's contributions, we would like to stop at the crossroad of ideas related to the complexity of many-body physics and the Weisskopf-Wigner damping theory. We consider a mesoscopic many-body system with internal dynamics governed by the mean field and residual two-body interactions. We assume that the system is perturbed by non-Hermitian terms in the Hamiltonian. These terms, in general, model an effective coupling to the external degrees of freedom such as the continuum of scattering states, radiation fields or measurements.

The subject of this work is cross-disciplinary and the approach implemented here is widely used in atomic, molecular and nuclear physics, see our recent work 1 and references therein. For a broader audience we structure the paper and conduct our discussion in a pedagogical manner. We start our work by presenting in Sec. 2 a well known description of the internal dynamics in an open many-body quantum system with the aid of the non-Hermitian and energy-dependent Hamiltonian. A simple example of

two interacting spins in the presence of decay is discussed in Sec. 3. Although this example has no complexity associated with the many-body dynamics, the important physical features related to decay, as well as mathematical aspects, are already there. This case serves as an introduction to our main discussion presented in Sec. 4 and based on the consideration of a system of interacting fermions coupled to a decay channel. Concluding remarks are given in Sec. 5 .

\section{Effective Hamiltonian of an open system}

The technique using a non-Hermitian and energy-dependent effective Hamiltonian is rather common and dates back to the Weisskopf-Wigner damping theory [2, works of Rice [3] and Fano 4], and Feshbach projection formalism [5]. It is assumed that the many-body states in the system can be separated into two classes, the internal (intrinsic) states and external ones. The set of internal states $|\alpha\rangle$ is generally understood to be composed of the states where all particles occupy bound mean-field orbitals. The fact that the system is open requires one to include into consideration a set of external many-body states. These states $|c ; E\rangle$ are viewed as decay or reaction states and contain particles in the continuum or radiation quanta; we label them with a continuous variable of energy and a set of other quantum numbers characteristic for a channel $c$. Discussion 
of the reactions is not our primary goal in this work, nevertheless we should emphasize Wigner's significant contribution to this subject [6, 7, 8].

Although there exist many different formulations of this technique, here we start from the Schrödinger equation

$$
H|\Psi\rangle=E|\Psi\rangle
$$

where the full wave function is a superposition of internal and external states,

$$
|\Psi\rangle=\sum_{\alpha} C_{\alpha}|\alpha\rangle+\sum_{c} \int d E \chi_{E}^{c}|c ; E\rangle .
$$

Eliminating the external states we come to the equation for the coefficients $C_{\beta}$ that describe the internal part of the wave function,

$$
\sum_{\beta}\left[\langle\alpha|H| \beta\rangle+\sum_{c} \int d E^{\prime} \frac{\left\langle\alpha|H| c ; E^{\prime}\right\rangle\left\langle c ; E^{\prime}|H| \beta\right\rangle}{E-E^{\prime}}-\delta_{\alpha \beta} E\right] C_{\beta}=0 .
$$

This equation looks like a traditional eigenvalue problem with the effective Hamiltonian matrix in the intrinsic space defined as

$$
\langle\alpha|\mathcal{H}(E)| \beta\rangle=\langle\alpha|H| \beta\rangle+\sum_{c} \int d E^{\prime} \frac{A_{\alpha}^{c}\left(E^{\prime}\right) A_{\beta}^{c}\left(E^{\prime}\right)^{*}}{E-E^{\prime}},
$$

where we introduced $A_{\alpha}^{c}(E)=\langle\alpha|H| c ; E\rangle$, the coupling amplitudes between internal and external states.

The first term in Eq. (4) is the standard Hamiltonian matrix that would describe

our system if it were decoupled from the external world. For definiteness, we assume that this part of the Hamiltonian is

$$
H=\sum_{1} \epsilon_{1} a_{1}^{\dagger} a_{1}+\frac{1}{4} \sum_{1234} V_{12 ; 34} a_{1}^{\dagger} a_{2}^{\dagger} a_{3} a_{4},
$$

with single-particle energies $\epsilon_{1}$ and residual two-body interactions with antisymmetrized matrix elements $V_{12 ; 34}$. The indexes $1, \ldots$, contain a complete set of single-particle quantum numbers, and the total many-body basis state $\alpha$ can be constructed, in the case of Fermi-statistics, as a Slater determinant of these single-particle states.

The integral in Eq. (4) can be further decomposed into its Hermitian part (principal value) and the remaining non-Hermitian part,

$$
\begin{gathered}
\sum_{c} \int d E^{\prime} \frac{A_{\alpha}^{c}\left(E^{\prime}\right) A_{\beta}^{c}\left(E^{\prime}\right)^{*}}{E-E^{\prime}}= \\
\sum_{c} P \int d E^{\prime} \frac{A_{\alpha}^{c}\left(E^{\prime}\right) A_{\beta}^{c}\left(E^{\prime}\right)^{*}}{E-E^{\prime}}-i \pi \sum_{c(\text { open })} A_{\alpha}^{c}(E) A_{\beta}^{c}(E)^{*} .
\end{gathered}
$$

Thus, the effective Hamiltonian (17) can be written as

$$
\mathcal{H}(E)=H+\Delta(E)-\frac{i}{2} W(E)
$$




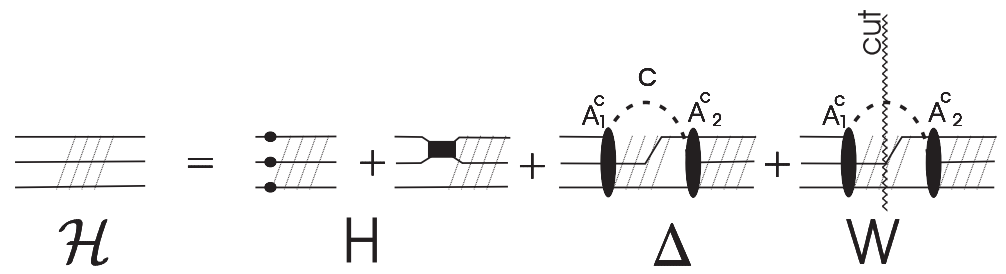

Figure 1. A schematic representation of the effective Hamiltonian of an open system. The Hermitian shell model part $H$ is shown by the two terms following Eq. (5), singleparticle energies and two-body residual interactions. The term $\Delta$ includes all channels $c$ while the term $(-i / 2) W$ is restricted to the channels open at a given energy.

which contains an energy-dependent self-energy term due to the "off-shell" processes of virtual excitation into all, open and closed at given energy, continuum channels,

$$
\langle\alpha|\Delta(E)| \beta\rangle=\sum_{c} P \int d E^{\prime} \frac{A_{\alpha}^{c}\left(E^{\prime}\right) A_{\beta}^{c}\left(E^{\prime}\right)^{*}}{E-E^{\prime}},
$$

and explicitly non-Hermitian and energy dependent term

$$
\langle\alpha|W(E)| \beta\rangle=2 \pi \sum_{c(\text { open })} A_{\alpha}^{c}(E) A_{\beta}^{c}(E)^{*} .
$$

The imaginary part $W$ has a factorized form, and originates from the real "on-shell" processes through the continuum channels that are open at given energy. The schematic diagrams representing various terms in (7) are shown in Fig. 1,

For our current discussion we will ignore the energy dependence of the Hamiltonian (71). In general, the energy dependence of $\Delta$ is smooth and frequently this Hermitian term can be incorporated into the intrinsic Hamiltonian $H$ as a renormalization. The energy dependence of the non-Hermitian part $W$ can be crucial, in particular near channel thresholds, see for example Wigner's work [8]. In loosely bound systems this term determines the exact binding energy [1. Here, however, we are going to discuss the new effects and especially the phase transition generated by the presence of nonHermitian terms in the Hamiltonian, and for this purpose the amplitudes $A_{\alpha}^{c}$ can be taken as energy-independent parameters, as it would take place in a physical situation with remote thresholds. We simply assume that the Hamiltonian $\mathcal{H}=H-i W / 2$ is known, the anti-Hermitian part has a factorized structure (9), and this will allow us to study the resulting dynamics.

\section{Two spin-system}

We start with a very simple example similar to the system discussed by Dicke [9] in relation to superradiance. Consider two interacting distinguishable spin- $1 / 2$ molecules, $s_{1}=s_{2}=s=1 / 2$, with the spin-spin interaction

$$
H^{\circ}=\alpha \vec{s}_{1} \cdot \vec{s}_{2} .
$$


This Hamiltonian is diagonal in the basis of the total spin $\vec{S}=\vec{s}_{1}+\vec{s}_{2}$ and its $z$-projection, and the energies of the states,

$$
E_{S}=\frac{\alpha}{2}[S(S+1)-2 s(s+1)],
$$

are $E_{0}=-3 \alpha / 4$ for the singlet, $S=0$, state and $E_{1}=\alpha / 4$ for the triplet, $S=1$, state.

Now let us place the system in the magnetic field that produces two effects. First, it leads to the Zeeman splitting that can be described by the additional term in the Hamiltonian

$$
H^{\mathrm{B}}=\epsilon s_{1}^{z}+\epsilon s_{2}^{z}=\epsilon S^{z} .
$$

For simplicity we assume that the field acts in the same way on both molecules, therefore the additional term (12) still commutes with the operator $\vec{S}^{2}$. Secondly, we assume that this two-spin system in the magnetic field becomes open; in the presence of the field the first molecule in its excited polarized state can dissociate, the phenomenon similar to the Feshbach resonance [10]. This means that the molecule in $s_{1}^{z}=1 / 2$ state decays exponentially with half-life $T_{1 / 2}$ which can be described by the width $\gamma=\ln 2 / T_{1 / 2}$. In terms of the general formalism of Sec. 2 here we have one open decay channel with coupling strength $A$ and $\gamma=A^{2}$. In principle the decay rate of a molecule should depend on the magnitude of the Zeeman splitting and thus on the field strength. For the interacting system the decay clearly should depend on total energy. As we stated earlier, we ignore this dependence in our examples. The situation can be modelled by an extra non-Hermitian term in the Hamiltonian

$$
W=-i \frac{\gamma}{4}\left(s_{1}^{z}+s\right)
$$

so that the first molecule in the state with $s_{1}^{z}=1 / 2$ would have decay width $\gamma$ and this width would be zero in the state $s_{1}^{z}=-1 / 2$. As a result, in the magnetic field the effective Hamiltonian for the two-spin system becomes

$$
\mathcal{H}=\alpha \vec{s}_{1} \cdot \vec{s}_{2}+\epsilon s_{1}^{z}+\epsilon s_{2}^{z}-i \frac{\gamma}{4}\left(s_{1}^{z}+s\right)
$$

The breakup of the first molecule distorts the symmetry of the system, $\vec{S}^{2}$ no longer commutes with $\mathcal{H}$, and the singlet and triplet states become mixed. The situation here is interesting in the sense that the open decay channel introduces an incompatible symmetry: the interaction Hamiltonian (11) and the magnetic field part (12) are both diagonal in the basis of the total spin $\left(S, S^{z}, s_{1}, s_{2}\right)$, whereas the decay part, as that of an intrinsic elementary process of the molecule 1 , is diagonal in the decoupled spin states of molecules $\left(s_{1}, s_{1}^{z} ; s_{2}, s_{2}^{z}\right)$, the so-called $m$-scheme basis. The axial symmetry with respect to the magnetic field holds for the full Hamiltonian (14) requiring the conservation of $S^{z}$.

The full effective Hamiltonian matrix of the $S^{z}=0$ block in the $m$-scheme basis takes the form

$$
\mathcal{H}=-\frac{\alpha}{4}+\frac{1}{2}\left(\begin{array}{cc}
-i \gamma & \alpha \\
\alpha & 0
\end{array}\right)
$$




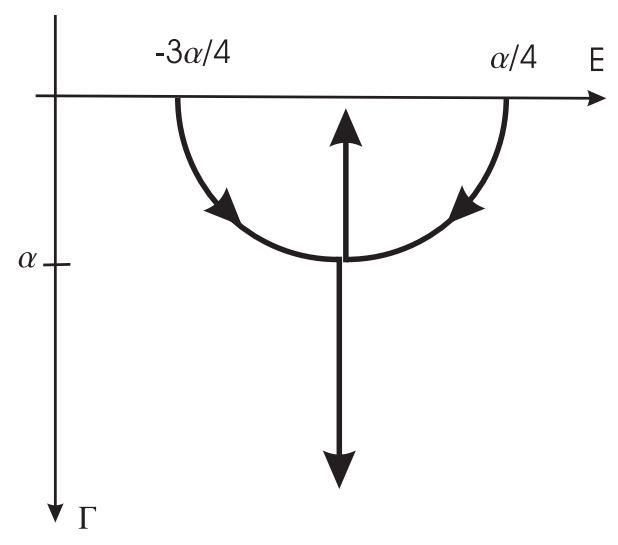

Figure 2. Evolution of two states $\mathcal{E}=E-i \Gamma / 2$ in the complex plane as a function of the increasing parameter $\gamma$.

and the diagonalization of this matrix determines the quasistationary eigenstates of the system and their complex energies,

$$
\mathcal{E}_{ \pm}=-\frac{\alpha}{4} \pm \frac{1}{2} \sqrt{\alpha^{2}-\left(\frac{\gamma}{2}\right)^{2}}-i \frac{\gamma}{4}
$$

The trajectories of the complex energies $\mathcal{E}_{ \pm}$as functions of the increasing decay parameter $\gamma$ are shown in Fig. 2,

The dynamics of the two-level effective Hamiltonians have been extensively studied in the past, see for example 11, 12, 13, 14]. In fact the discussion was initiated by Wigner and von Neumann [15], who demonstrated that mixing by an off-diagonal Hermitian interaction leads to the level repulsion. The behavior of complex eigenstates as a function of real and complex parts of the effective Hamiltonian can be summarized as follows. It is well known that the increase of the Hermitian part of the interaction leads to repulsion of real energies $E$ and attraction of widths $\Gamma$ since the decay probability is shared by the two mixed states. It is less well known that the non-Hermitian part has an opposite effect, it increases the difference between widths and moves real parts $E$ closer together [1, 12, 14; these effects are schematically demonstrated in Fig. 3] 12.

Our example reveals the generic features, consider Eq. (16) and Fig. 2, In the region of weak decay, i.e. slow process of molecular disintegration, $0 \leq \gamma \leq 2 \alpha$, the two eigenstates $\mathcal{E}_{+}$and $\mathcal{E}_{-}$have equal widths, $\Gamma_{+}=\Gamma_{-}=\gamma / 2$, and the real energies $E_{+}$and $E_{-}$are attracted to each other as $\gamma$ grows. At the critical point $\gamma=2 \alpha$ the levels cross and the "phase transition" occurs as a sharp change in the behavior of the two complex energies. The real parts become degenerate, $E_{+}=E_{-}=-\alpha / 4$, while the widths separate, $\Gamma_{ \pm}=\gamma / 2 \pm \sqrt{(\gamma / 2)^{2}-\alpha^{2}}$ so that in the limit of strong decay, $\gamma \gg \alpha$, we have a single "superradiant" state with the width $\Gamma_{+} \rightarrow \gamma$ accumulating the entire available decay probability, and one long-lived state with $\Gamma_{-} \rightarrow 0$. The factorized structure of matrix $W$ (92), that results in zero eigenvalues in the limit when this part dominates the Hamiltonian, is a key feature responsible for this behavior. As can be seen from the derivation of Sec. 2, this structure is related to the analytical structure of the problem 


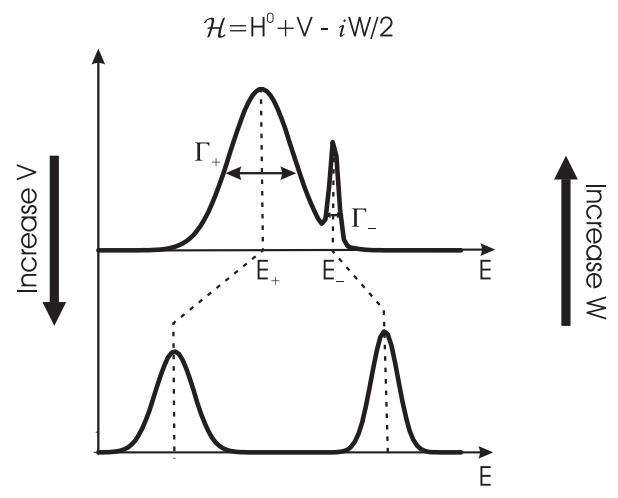

Figure 3. Generic behavior of two resonances $E_{ \pm}$and their widths $\Gamma_{ \pm}$as a result of increase of the Hermitian part of mixing interaction $V$ and in response to increase of non-Hermitian mixing $W$.

and unitarity requirements. The formation of fast and slow decay modes, which can be viewed as an ultimate width repulsion, is a typical effect in systems with overlapping resonances [16, 17]. In particular, a detailed discussion for two-level examples can be found in 11, 13, 14, 18, and references therein.

The coupling to decay, as we see, has important observable consequences for both the intrinsic state of the system and the external scattering picture. For example, for a singlet ground state $(\alpha>0)$, the magnetic field does not affect the system in the absence of molecular breakup, because $H_{\mathrm{B}}$ is identically zero in the singlet state. In the presence of the field, the disintegration of the first molecule reorients the eigenvector of the system admixing a triplet state. Thus, the system becomes susceptible to an excitation by the magnetic pulse.

Looking at the situation from a different angle, namely from the "exterior", it is clear that the decay of a first molecule alone is different from the decay properties when the second molecule is present. In the first paper on superradiation [9] Dicke argued that coupling to the radiation field can lead to strong modification of the intrinsic properties of the system. In context of nuclear reaction theory, a significant contribution to this subject was made by Wigner and his collaborators who discussed the nature of resonances in the nucleon-nucleus interactions, sum rules related to the widths, distribution of the widths among the states and general features of the scattering cross section [6, 7, 19], that in context of our example can be related to the properties of the matrix (15).

\section{A model of an open many-body system}

The transition to superradiance in a many-body model with decay and random internal dynamics is our second example. We consider an equidistant set of $\Omega$ fermionic singleparticle levels ("orbitals") and assume that the upper level, that we label as $\nu$, belongs to the continuum, i.e., in the approximation of non-interacting particles, the single-particle 


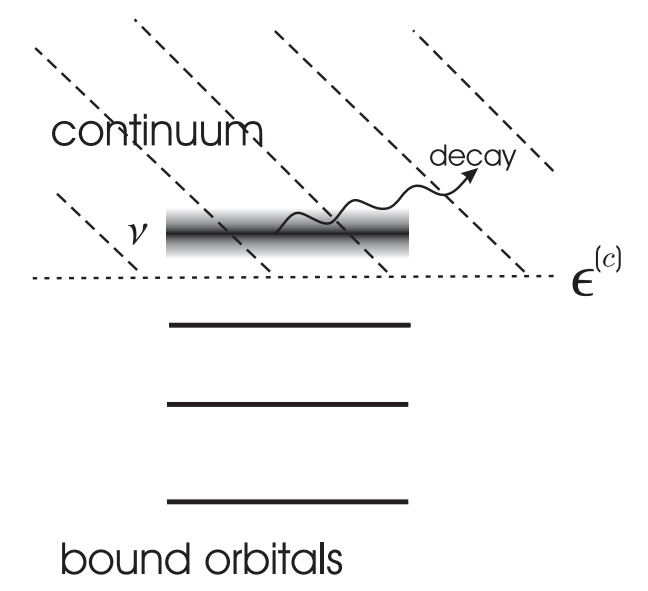

Figure 4. Schematic drawing of the single-particle states in the model of Sec. 4 The upper state belongs to the continuum.

wave function of this orbital decreases exponentially with time, while the remaining orbitals are particle-stable, see Fig. 4. This situation again corresponds to a single decay channel where any many-body state with an occupied single-particle orbital $\nu$ is coupled to the continuum with the corresponding amplitude $A$. Equivalently the full non-Hermitian effective Hamiltonian $\mathcal{H}$ can be constructed from (5) under assumption that one of the single-particle energies is complex,

$$
e_{\nu}=\epsilon_{\nu}-\frac{i}{2} \gamma, \quad \gamma=A^{2}
$$

it contains single-particle energies with the scale determined by their spacing $\Delta \epsilon$, the non-Hermitian part $W$, the scale of which is determined by $\gamma$, and the Hermitian mixing $V$, Eq. (5). We do not impose any symmetry here; the effects of Kramers degeneracy and rotational invariance have been discussed in [1]. For our example we select the parameters of the Hermitian residual two-body interaction $V$, eq. (5) at random from the Gaussian distribution centered at zero. The average absolute magnitude of these

matrix elements $\sqrt{\overline{V^{2}}}$ sets the energy scale for the model. In what follows we assume $\sqrt{\overline{V^{2}}}=1$ and allow $\Delta \epsilon$ and $\gamma$ to vary.

\subsection{Evolution of many-body states}

A system of $N$ fermions in $\Omega$ single-particle states has

$$
\mathcal{N}=\frac{\Omega !}{N !(\Omega-N) !}
$$

many-body states. To be specific, we consider $N=4$ particles in $\Omega=8$ single-particle orbitals. The $\mathcal{N}=70$ eigenvalues $\mathcal{E}_{j}=E_{j}-(i / 2) \Gamma_{j}$ of the Hamiltonian are in the lower part of the complex plane, and their motion as a function of the increasing parameter $\gamma$ is shown in Fig. 5, here $\Delta \epsilon=1$. This figure is a generalization of the situation in Fig. 2] discussed in the previous section 3. As stated earlier, it is assumed that $\mathcal{H}$ is 
independent of energy $E$. Therefore the trace conservation leads to a helpful sum rule for the imaginary part of the Hamiltonian,

$$
\sum_{j} \Gamma_{j}(\gamma)=-2 \operatorname{Im}(\operatorname{Tr} \mathcal{H}) .
$$

This sum rule is a direct analog to the sum rules discussed by Wigner and collaborators in the context of neutron resonances [6].

Several dynamical limits can be identified. In the "shell-model" limit of $\gamma=0$ and $\mathcal{H}=H$, standard diagonalization results in orthonormal eigenstates $\Psi_{\text {s.m. }}$ with real

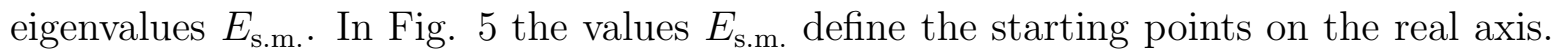
As soon as the single-particle width $\gamma \neq 0$, the decay channels open. The random residual interaction $V$ leads to a chaotic mixing of configurations, and all many-body states acquire widths. This excludes special cases when configurations with the occupied decaying state are blocked, for example by selection rules.

For small $\gamma$, the term $W$ in the Hamiltonian can be treated perturbatively. In the lowest order this results in

$$
\mathcal{E}=E_{\mathrm{s} . \mathrm{m} .}-\frac{i}{2}\left\langle\Psi_{\mathrm{s} . \mathrm{m}}|W| \Psi_{\mathrm{s} . \mathrm{m}}\right\rangle .
$$

Then the decay width of a many-body state is determined by the occupancy $n_{\nu}(\Psi)=$ $\left\langle\Psi_{\mathrm{s} . \mathrm{m}}\left|a_{\nu}^{\dagger} a_{\nu}\right| \Psi_{\mathrm{s} . \mathrm{m}}\right\rangle$ of a decaying orbital $\nu$ calculated for a particular shell model state $\Psi_{\text {s.m. }}$,

$$
\Gamma(\Psi)=\left\langle\Psi_{\mathrm{s} . \mathrm{m}}|W| \Psi_{\mathrm{s} . \mathrm{m}}\right\rangle=\gamma n_{\nu}\left(\Psi_{\mathrm{s} . \mathrm{m} .}\right) .
$$

Only in this limit it is possible to establish the "natural" result (21) that is widely used in extracting spectroscopic factors of intrinsic states from various reaction cross sections.

The opposite limit of strong $\gamma$ is less trivial. It is clear from Fig. 1 that all manybody states are divided into two groups. In the first group the complex energies have a rapidly increasing width, whereas the states in the second group become long-lived with their widths approaching zero. The dominance of the external coupling $W$ is the origin of this dynamical separation. The single-particle energies and mixing $V$ are of minor importance in this limit, and the states can be classified by their relation to the decay. The matrix $W$ is highly degenerate; because of its factorized structure, Eq. (9), it has only

$$
\mathcal{N}_{\Gamma}=\frac{(\Omega-1) !}{(N-1) !(\Omega-N) !}
$$

nonzero eigenvalues. The corresponding eigenstates of $W$ are the configurations that have the decaying upper orbital occupied and thus acquire in the strong decay limit the maximum width equal to $\gamma$ so that the trace (19) of the imaginary part of the Hamiltonian is exhausted by $\gamma \mathcal{N}_{\Gamma}$. In our case, the total dimension of the many-body space is $\mathcal{N}=70$, and $\mathcal{N}_{\Gamma}=35$. The remaining 35 states correspond to the empty upper orbital, and correspondingly to the zero eigenvalues of $W$, so that decay of these states is strongly hindered. 


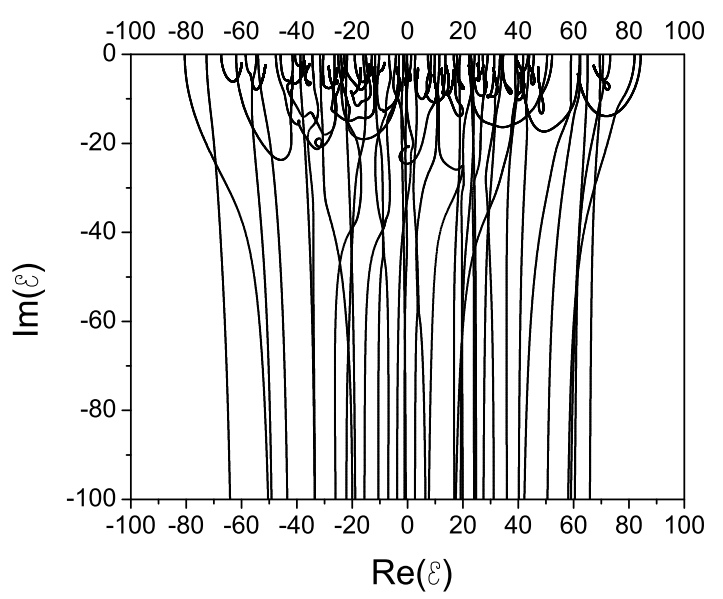

Figure 5. Complex plane trajectories of all 70 many-body states in the system of 4 particles in 8 equidistant single-particle levels with the spacing of $\Delta \epsilon=0.5$ energy units as a function of the decay width $\gamma$ of the upper single-particle state increasing downwards. The two-body interaction matrix elements are taken randomly from the Gaussian distribution with zero mean and variance of one energy unit.

Thus, in the limit of strong coupling to the continuum, a dynamical segregation of short-lived and long-lived (compound) intrinsic states occurs. This is a generalization of the effect observed in a simple two-level system of Sec. 3. This phenomenon was discovered in numerical simulations [20, 21], see also [22, 23], was studied in molecular and atomic physics [24, 25, 26] and observed in experiments with microwave cavities 27. The fact that the underlying physics is analogous to the Dicke coherent state [9] was explained in Refs. [16, 17]. The coupling of states via a common decay channel (width collectivization) plays a role similar to that of the common radiation field of spontaneously radiating atoms for the Dicke superradiant coherent mode experimentally observed in quantum optics [28, 29].

A related effect considered in the quantum theory of measurements has possible ramifications for quantum computers. An external influence on the system brought by a procedure of measurement, the Zeno effect [30], can be modelled similarly, with the aid of non-Hermitian terms in the Hamiltonian. Recently this topic attracted a lot of attention due to the appearance of remarkable experimental data [30, 31, 32. In this context it was demonstrated by Facchi and Pascazio [33] that an analogous effective subspace separation plays a vital role. We need to emphasize that the dominating term in the Hamiltonian, $W$, because of its degeneracy, only effectively decouples the subspaces but it does not fully determine the dynamics. The single-particle energies and residual Hermitian interactions determine the motion within the intrinsic subspace that still can be chaotic.

The situation at intermediate values of the decay strength, as seen from Fig. [ is complicated. In this region all three terms of the effective Hamiltonian are important, 
and their interplay is responsible for chaotic transitional dynamics. As $\gamma$ increases, the perturbative approach breaks down. However, at this point the limit of the full subspace decoupling is not yet reached. In this regime we are faced with a peculiar phase crossover, where all components of the dynamics are strongly mixed and thus both energy centroids $E_{j}$ and widths $\Gamma_{j}$ become affected in a non-trivial way by the presence of the non-Hermitian component. A similar interplay between single-particle energies and the residual pairing interaction is known to result in chaotic dynamics of the normal-to-superconducting phase transition in mesoscopic systems 34] which, due to the finite nature of the system, occurs as an extended crossover.

In his work related to nucleon-nucleus interaction [6], Wigner emphasized the importance of interplay between the state of intrinsic configuration (determined by Hermitian interaction $V$ ) and doorway states (the eigenstates of $W$ ). He pointed out that three types of behavior can be distinguished by the distribution of the widths among many-body states. In the limit of $V \rightarrow 0$, the "independent particle model," the entire width is absorbed by the set of states where the decaying single-particle orbital is fully occupied. This is an analog to the superradiant regime. In the intermediate regime, due to the interaction $V$, the width becomes fragmented and spreads to many states. Finally, in the limit of large $V$ the chaotic intrinsic motion leads to the approximately uniform distribution of the widths, the "uniform model," which is analogous here to the shell-model limit $\gamma \rightarrow 0$.

\subsection{Effective occupation numbers}

We can extend the definition of the single-particle occupancy $n_{\nu}$ of the decaying orbital to any value of $\gamma$, instead of the $\gamma \rightarrow 0$ limit discussed previously, eq. (21). The effective occupation number for the quasistationary state $j$ can be defined as

$$
n_{\nu}(j ; \gamma)=\frac{\partial \Gamma_{j}(\gamma)}{\partial \gamma}
$$

As follows from Eq. (19), the sum $\sum_{j} n_{\nu}(j ; \gamma)$ is independent of $\gamma$. The numbers (23), properly normalized according to Eq. (19), show how additional decay amplitudes induced by an infinitesimal increase of $\gamma$ are distributed among the widths of the eigenstates. Despite the resemblance to occupation numbers, these $n_{\nu}$ can be negative and do not have to be confined to the interval between 0 and 1 . There exists also another possibility, namely, to introduce spectroscopic factors directly as $\Gamma_{j}(\gamma) / \gamma$. They are indeed between 0 and 1 , but being simply proportional to $\Gamma$ they do not reveal any new information related to the dynamics.

In Fig. [6 the evolution of the effective occupation numbers $n_{\nu}$ for the lowest 13 states $\Psi_{j}$ is shown. The evolution starts with occupancies found for the shell model with residual interaction but no decay and follows a complicated path to the final segregated values of 0 and 1, corresponding to quasistable and superradiant states, respectively. The resulting crossover transition of the entire spectrum is gradual. It starts at the point where $\gamma$ exceeds the level spacing on the real axis. Here the resonances start 


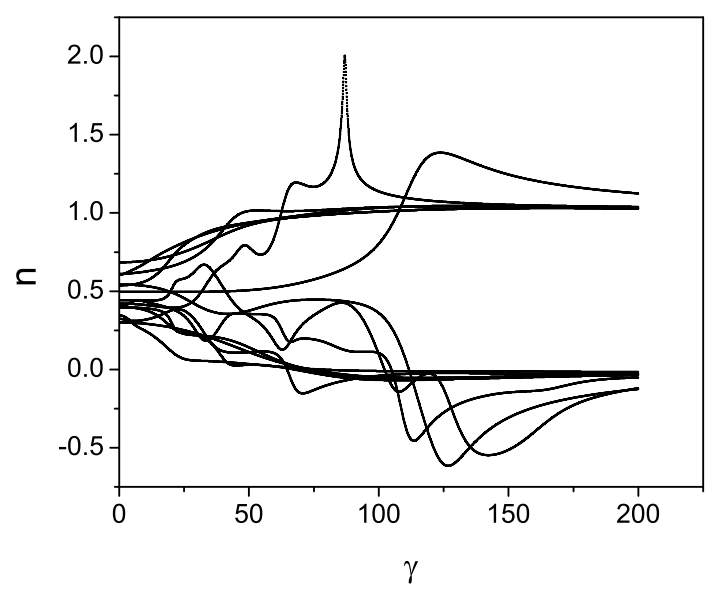

Figure 6. The evolution of the effective occupation numbers $n_{\nu}$, Eq. (23), as a function of $\gamma$ for the 13 lowest (selected at $\gamma=0$ ) many-body states.

to overlap. Although there are states that converge to the limiting value relatively fast, the majority of states undergo a complicated and peculiar evolution driven by the competition between the intrinsic and external interactions. Before reaching the regime of developed segregation, the evolution may involve peaks of occupation numbers for specific eigenstates. The segregation occurs at the values of width covering the macroscopic portion of the many-body spectrum, so that a balance between the external coupling represented by the imaginary part and the internal motion given by Hermitian part is reached. An analogous balance between two-level atoms (radiators) and radiation field is required for the Dicke superradiance. The nature of the phase transition to superradiance in the Dicke model was studied in Refs. [35, 36].

The definition of the effective occupation numbers in the form (23) provides a special insight into the dynamics of a phase transition. This definition is similar in spirit to that of correlational entropy [37] that shows how fast the states of the system readjust as a result of changes in the environment. Here $\gamma$ plays the role of the external parameter. For regular dynamics the behavior of this entropy is smooth. However, any phase transition, which may be hindered in a mesoscopic system, leads to a rapid restructuring of the state and thus results in a peak in the correlational entropy [34, 38]. Similarly, in our example, in the transitional region of $\gamma$, the peaks of $n_{\nu}$ as a function of $\gamma$ are clearly seen for most of the states $\Psi_{j}$. For some states in Fig. 2 the peaks can be sharp, an indication that a particular many-body state undergoes a sudden restructuring. It is worth noting that a practically identical discussion is appropriate for the two-spin example considered in Sec. 3. There the quantity $n_{\nu}$ becomes the effective probability of finding the molecule 1 in the radiating state with $s^{z}=1 / 2$. For the two mixed states with $S^{z}=0$ it can be easily calculated using the definition (23) and the expression for the complex energy (16). Even in the limited example of two 


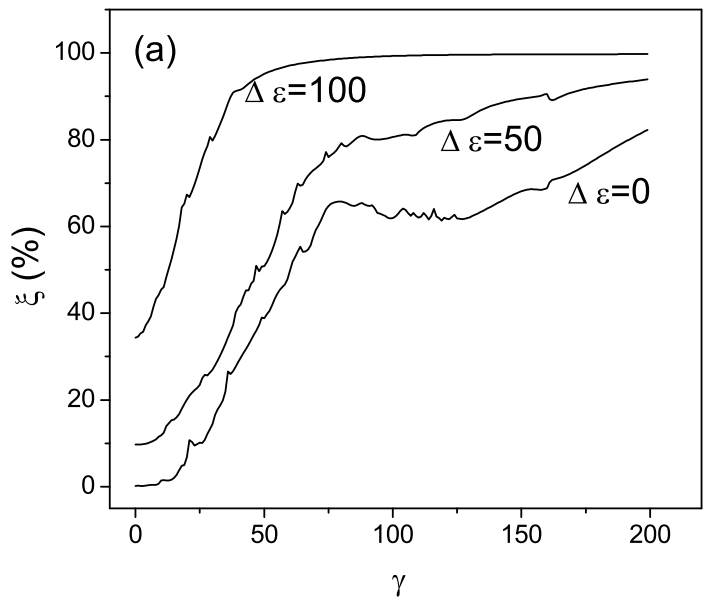

Figure 7. Fraction of segregated states as a function of $\gamma$. The single-particle level spacing is varied from the degenerate case, $\Delta \epsilon=0$, to $\Delta \epsilon=100$, a point where the residual interaction $V$ can be completely ignored.

spins the "phase transition" (the point of level crossing) appears as a special bifurcation point.

\subsection{Global transition to superradiance}

In Fig. 7 we demonstrate the global properties of the transitional regime from another perspective by plotting the segregation fraction $\xi(\gamma)$ defined as a fraction of many-body states for which the generalized occupancy $n_{\nu}$ appears close to the limiting values of 0 or 1 ,

$$
\xi(\gamma)=\frac{1}{\mathcal{N}} \sum_{j}\left[e^{-n_{\nu}^{2}(j ; \gamma) /\left(2 \sigma^{2}\right)}+e^{-\left[1-n_{\nu}^{2}(j ; \gamma)\right]^{2} /\left(2 \sigma^{2}\right)}\right] .
$$

Here we choose $\sigma=0.1$. The role of the relative magnitudes of the single-particle spacing $\Delta \epsilon$ and two-body matrix elements for the phase transition can be inferred from this figure. Here a randomly chosen set of two-body matrix elements $V$ with an average magnitude setting the unit of energy is kept constant, while the single-particle level spacing $\Delta \epsilon$ is varied. In the limit of infinitely large spacing, the mixing part of the Hamiltonian, $V$, becomes negligible, formally resulting in the disappearance of the intermediate transitional region. Nevertheless, even for $\Delta \epsilon$ fifty times larger than the average $V$, the transitional region is still well defined.

The transition to superradiance, being gradual for the entire system, can be very drastic for a particular many-body state. An interesting analogy can be found between the transition to superradiance and percolation theory. The set of original intrinsic states can be considered as a lattice with cites determined by energies $E_{\mathrm{s} . \mathrm{m}}$. Without decay the system is stationary and rests at a given initial eigenstate. The coupling to 
the continuum opens the possibility for the system to hop from state to state until it gets into a doorway state and emits a particle. The probability of such hopping $j \rightarrow j^{\prime}$ according to the perturbation theory is determined by the matrix element $\left\langle j|W| j^{\prime}\right\rangle$ (this term in our examples is proportional to $\gamma$ ) and inversely proportional to the distance $\left|E_{\mathrm{s} . \mathrm{m} . j}-E_{\mathrm{s} . \mathrm{m} \cdot j^{\prime}}\right|$. The lattice is irregular because the cites of the lattice correspond to the eigenvalues of the Hamiltonian $H$ and the distribution of distances between the cites is given by Wigner random matrix theory [39]. As a result, some areas can experience phase transitions and coherently orient toward a doorway state (which is not an eigenstate) earlier than the rest of the system. This is the reason for the sharp changes observed in individual states, see Fig. [6 and related discussion. As follows from Fig. 7. a significant strength of the continuum coupling is required for the full system to reorient and to create a coherent superradiant flow into doorway configurations.

\section{Conclusions}

We presented an introduction to interesting phenomena that appear in complex systems as a result of their coupling to the environment. We discussed simple examples showing that the strong coupling significantly reorganizes the motion, dynamically separating the states by their relation to the external world. We investigated the properties of a manybody phase transition from the normal shell model (or Fermi-liquid) behavior of a closed many-body system to the superradiant regime with the segregation of rapidly decaying states from long-lived resonances. The chaotic character of the intrinsic interaction does not prevent the system from this generic transition. The presence of very longlived states, that are almost decoupled from the external world, maybe important for the problem of quantum computing.

We deliberately related our examples to different branches of physics and provided many references trying to demonstrate that these phenomena can be relevant to almost any case when the coupling of a system to the outside world cannot be neglected. This topic links together many of the ideas inherited from Wigner. They include the complexity of many-body states with the characteristic level repulsion [15]; the theory of quantum damping and decay [2]; the role of symmetry and incompatibility between symmetries represented here by the competition between internal symmetry and symmetry imposed by the decay processes; theory of resonances, resonance sum rules, fragmentation of the widths in reactions [6, 7, 8] and the near-threshold width behavior [8]; random matrix theory [39].

\section{Acknowledgments}

This work was supported by the U. S. Department of Energy, Nuclear Physics Division, under contract No. W-31-109-ENG-38, and by the National Science Foundation, grant PHY-0070911. 
[1] Volya A and Zelevinsky V 2002 Argonne National Laboratory preprint PHY-10327-TH-2002, LANL preprint nucl-th/0211039

[2] Weisskopf V and Wigner E 1930 Z. Phys. 63 54; 19306518.

[3] Rice O 1933 J. Chem. Phys. 1375.

[4] Fano U 1935 Nuovo Cim. 12 156; 1961 Phys. Rev. 1241866.

[5] Feshbach H 1958 Ann. Phys. 5 357; 196219287.

[6] Lane A, Thomas R and Wigner E 1955 Phys. Rev. 98693.

[7] Teichmann T and Wigner E 1952 Phys. Rev. 87123.

[8] Wigner E 1948 Phys. Rev. 731002.

[9] Dicke R 1954 Phys. Rev. 9399.

[10] Stwalley W 1976 Phys. Rev. Lett. 371628.

[11] Sokolov V V and von Brentano P 1994 Nucl. Phys. A578 134.

[12] von Brentano P 1996 Phys. Rep. 26457.

[13] Philipp M et al 2000 Phys. Rev. E 621922.

[14] von Brentano P, Jolos R and Weidenmüller H 2002 Phys. Lett. B 53463.

[15] Von Neumann J and Wigner E 1929 Phys. Z. 30467.

[16] Sokolov V V and Zelevinsky V 1988 Phys. Lett. B 20210.

[17] Sokolov V V and Zelevinsky V 1989 Nucl. Phys. A504 562.

[18] Desouter-Lecomte M and Jacques V 1995 J. Phys. B 283225.

[19] Teichmann T 1050 Phys. Rev. 77506.

[20] Moldauer P 1975 Phys. Rev. C 11426.

[21] Kleinwachter P and Rotter I 1985 Phys. Rev. C 321742.

[22] Haake F et al 1994 Z. Phys. B 88359.

[23] Persson E and Rotter I 1999 Phys. Rev. C 59164.

[24] Pavlov-Verevkin V 1988 Phys. Rev. A 129168.

[25] Coleman P and Knight P 1982 J. Phys. B 15 L235.

[26] Knight P, Lauder M and Dalton B 1990 Phys. Rep. 1901.

[27] Persson E et al 2000 Phys. Rev. Lett. 852478.

[28] Skribanowitz N et al 1973 Phys. Rev. Lett. 30309.

[29] Gross M et al 1976 Phys. Rev. Lett. 361035.

[30] Kofman A and Kurizki G 2000 Nature 405546.

[31] Kofman A and Kurizki G 2001 Phys. Rev. Lett. 87270405.

[32] Fischer M, Gutierrez-Medina B and Raizen M 2001 Phys. Rev. Lett. 87040402.

[33] Facchi P and Pascazio S 2002 Phys. Rev. Lett. 89080401.

[34] Volya A, Zelevinsky V and Brown B A 2002 Phys. Rev. C 65054312.

[35] Wang Y and Hioe F 1973 Phys. Rev. A 7831.

[36] Hioe F 1973 Phys. Rev. A 81440.

[37] Sokolov V V, Brown B A and Zelevinsky V 1998 Phys. Rev. E 5856.

[38] Cejnar P, Zelevinsky V and Sokolov V V 2001 Phys. Rev. E 63036127.

[39] Wigner E 1955 Ann. Math. 62 548; 195765 203; 195867325. 